\title{
Characteristics Analysis and Modeling of Fault Sound and Background Noise of Large Central Air Conditioner
}

\author{
Li Junhao", Liu Wenhong, ${ }^{1, \text {, * }}$ \\ ${ }^{1}$ School of Electrical Engineering, Shanghai Dianji University, Shanghai, China \\ ${ }^{2}$ School of Electronic Information, Shanghai Dianji University, Shanghai, China \\ Email address: \\ liuwenhong@sdju.edu.cn (Liu Wenhong),1273889241@qq.com (Li Junhao) \\ ${ }^{*}$ Corresponding author
}

\section{To cite this article:}

Li Junhao, Liu Wenhong. Characteristics Analysis and Modeling of Fault Sound and Background Noise of Large Central Air Conditioner. Journal of Electrical and Electronic Engineering. Vol. 6, No. 1, 2018, pp. 20-25. doi: 10.11648/j.jeee.20180601.14

Received: January 28, 2018; Accepted: February 13, 2018; Published: March 9, 2018

\begin{abstract}
Performance of time delay estimation (TDE) algorithm directly affects effect of electromechanical equipment fault sound source positioning based on TDE. Designing more accurate algorithm based proper models of background noise and fault sound can improve accuracy of localization. Firstly, this paper analyzes the characteristics of noise and fault sound of external central air conditioners, then, models environmental noise using Alpha distribution. Finally, Analyzes measured data and preprocess them, realize the more appropriate modeling of environmental noise and eliminate operating noise sources interference. This study has a guiding significance in large-scale mechanical and electrical equipment failure sound source signal modeling, and is helpful to research on TDE algorithm.
\end{abstract}

Keywords: Electrical and Mechanical Equipment, Sound Source Positioning, TDE, Fault Sound Analysis, Noise Analysis and Process

\section{Introduction}

Mechanical and electrical equipment fault sound source localization based on TDE can determine Fault location, then posing solutions to fault, aiming to avoid fault to expand, reduce risk of accidents and decrease maintenance costs. Such as generator acoustic fault detection, [1] wind turbines source positioning, [2] power station boiler leakage source positioning. [3] Sound source positioning is in two stages to complete, TDE and geometric positioning. Performance of TDE has a direct impact on positioning effect. Actual fault sound signal collected always contains background noise, It is a sum of various sounds during operation removing fault sound signal. Modeling background noise and fault sound accurate model, then, designing suitable TDE algorithm can improve accuracy of TDE according to these signal models.

Gaussian distribution is used to model noise in many source localization based on TDE, [4] that accords with the process of noise generation, central limit theorem can prove its rationality. However, there are non-Gaussian noise with impulsive signals in the actual environment of mechanical and electrical equipment, such as low-frequency atmospheric noise, [5] mechanical and electrical equipment operation noise and man-made noise. [6] Gaussian-based noise is not suitable for describing noise with impulsive signal, it causes performance degradation of TDE Algorithm based on Gaussian assumption. Noise model can be approximated to a Gaussian distribution by blanking impulse noise means, [7] but other algorithm needs to be designed to get blanking noise threshold, it can affect signal characteristics in non-Gaussian noise with more impulsive signals.

This paper analyzes fault sound and operating noise of central air-conditioning machine, and theoretically analyzes electromechanical equipment environmental noise, uses Alpha distribution model equipment ambient noise and gives methods to determine noise model parameters. Then, acquire central air condition noise in operation, analyze noise characteristics in the time domain and frequency domain, and preprocess using corresponding frequency notch filter. 


\section{Fault Sound and Background Noise Analysis and Modeling}

\subsection{Fault Sound Analysis}

Air conditioner outside machine noisemainly includes:[8] abnormal noise caused by unmatched fan and air duct, AC noise generated by motor operation, low frequency noise caused by unreasonable pipeline design and abnormal noise caused by improper assembly process control, The noise generated by the machine resonance. Ref. [9] introduced 2.38 $\mathrm{kHz}$ fault howl in the access section of the refrigerant pipe.

Electromechanical equipment fault sound signal and voice signal, short-term energy sound signal is very different. Voice signal is non-stationary, time-varying; short-term energy sound signals such as gun sound, broken glass sound is short time, instantaneous energy; and mechanical and electrical equipment fault sound signal and background noise has a stable, fixed fault audio frequency band characteristics, low signal to noise ratio(SNR), and background noise is complex. They use same method to locate sound source, but signal processing methods are very different among them.

\subsection{Background Noise Analysis}

Background noise is a sum of various sounds during operation removing fault sound signal, it is divided into operational noise and environmental noise for analysis and modeling.

\subsubsection{Operating Noise Analysis}

Air conditioning running noise are mainly include: [8]fan operation airflow sound, compressor operation noise, refrigerant flow sound, pipeline vibration noise, air conditioning heat exchanger system operation noise. Noise generated by fan is between 500 and $800 \mathrm{~Hz}$, noise generated by compressor is between 1 and $2 \mathrm{kHz}$, noise of structure is about $100 \mathrm{~Hz}$, and low frequency noise is about 20-60 Hz.

Operational noise can be removed by overlay method, [10] but some components can't use it. It can be eliminated in the frequency domain through spectrum analysis, designing suitable parameter notch filter or band-elimination filter to get the remaining band noise data, then, signal processing, locating fault sound source. If fault signal overlaps with the frequency spectrum of normal frequency band, needing to use other methods to filter the operating noise.

\subsubsection{Environmental Noise Analysis}

Environment noise of air conditioner is non-Gaussian noise containing implusive signal, it may come from Atmospheric discharge;[11] Medium and high frequency electrical equipment switching process; mechanically operated and transmitted;[12] some artificial noise; Signal acquisition and conversion process.

\subsection{Alpha Stable Distribution}

Alpha stable distribution is a generalized Gaussian model. According to the generalized central limit theorem, [13] it is the only limit distribution that forms the sum of independent and identically distributed random variables, and Gaussian distribution is a subclass. The difference between Gaussian distribution and Alpha distribution is that Gaussian distribution has an exponential trailing and Alpha distribution has an algebraic trailing. Therefore, Alpha distribution better describes pulse process in noise.

There is no analytical expression for the probability density function of Alpha's stable distribution except only a few. The eigenfunction and the probability density function are the only deterministic relations with each other. The eigenfunction is essentially the inverse Fourier transform of the probability density function. Both of them can completely describe the statistical characteristics of a random distribution. The following is the introduction of the eigenfunction of the Alpha steady state distribution.

If random variable has parameters $0<\alpha \leq 2, \gamma \geq 0$, $-1 \leq \beta \leq 1$, and the real number $\mathrm{a}$, the eigenfunction has the following expression:

$$
\begin{aligned}
& \emptyset(\mathrm{t})=\exp \left\{j a t-\gamma|\mathrm{t}|^{\alpha}[1+\mathrm{j} \beta \operatorname{sgn}(\mathrm{t}) \omega(\mathrm{t}, \alpha)]\right\} \\
& \omega(\mathrm{t}, \alpha)= \begin{cases}\tan \frac{\alpha \pi}{2}, & \alpha \neq 1 \\
\frac{2}{\pi} \log |\mathrm{t}|, & \alpha=1\end{cases} \\
& \operatorname{sgn}(\mathrm{t})= \begin{cases}1, & \mathrm{t}>0 \\
0, & \mathrm{t}=0 \\
-1, & \mathrm{t}<0\end{cases}
\end{aligned}
$$

the random variable $\mathrm{X}$ obeys $\alpha$ stable distribution.

Parameter $\alpha$ is characteristic index in formula (1), it determines pulse degree of $\alpha$ stable distribution. The smaller $\alpha$ value is, the thicker tailing distribution is, and the more impulsive sample is. On the contrary, the $\alpha$ value becomes larger and the tailing of distribution becomes thinner and the impulsiveness of sample weakens. When $\alpha=2$, the $\alpha$ distribution corresponds to Gaussian distribution, and the $\alpha$ steady distribution is generalized Gaussian distribution. Parameter $\beta$ determines the slope of distribution, $\gamma$ is the dispersion coefficient, which is a measure of the degree of dispersion relative to the mean, and $a$ is the median or mean of alpha distribution. These parameters in the eigenfunction are determined from measured samples according to the methods in [14], [15] and [16].

In noise modeling research, computer needs to produce alpha steady-state distribution sample. Chambers, Mallows and Stuck et al. proposed an accurate and practical sample generation method. The basic idea is to make a series of independent non-uniform distribution random variables Linear transformation into a stable distribution of random variables. [17]

\subsection{SNR}

SNR is also one of the main signal characteristics in signal modeling process. There is no variance in Alpha stable distribution, which can't be measured by classical power 
definition, and can be measured by the dispersion coefficient $\gamma,[18]$ using mixed SNR instead, $\operatorname{SNR}=10 \log \left(\sigma^{2} / \gamma\right), \sigma^{2}$ is variance of acoustic signal. In calculation of actual environmental power, power can be expressed by taking the variance of the sample. In order to obtain electromechanical equipment background environment power, using relevant measuring instruments such as sound level meters determined the SNR within a specific range. [19]

\section{Measured Data Analysis and Preprocess}

\subsection{Background Noise Acquisition and Analysis}

Select central air-conditioning unit in work of Shanghai Dianji University Lingang Campus Gymnasium as the mechanical and electrical equipment background noise collection object, noise collection environment as show in Figure 1. According to [20], select $48 \mathrm{kHz}$ sampling frequency according to the frequency response range of stationary microphone, it can meet the highest frequency signal sampling requirement and eliminate aliasing of spectrum. Use wavrecord in MATLAB to start sound collection, collect background noise when air conditioner is working normally, record 480000 point, 16-bit resolution array.

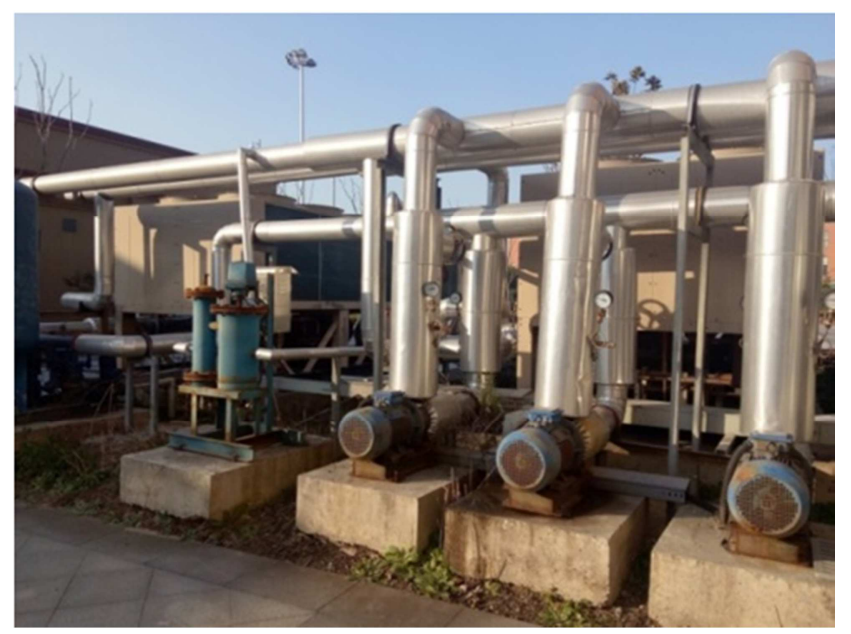

Figure 1. Central air conditioning environment.

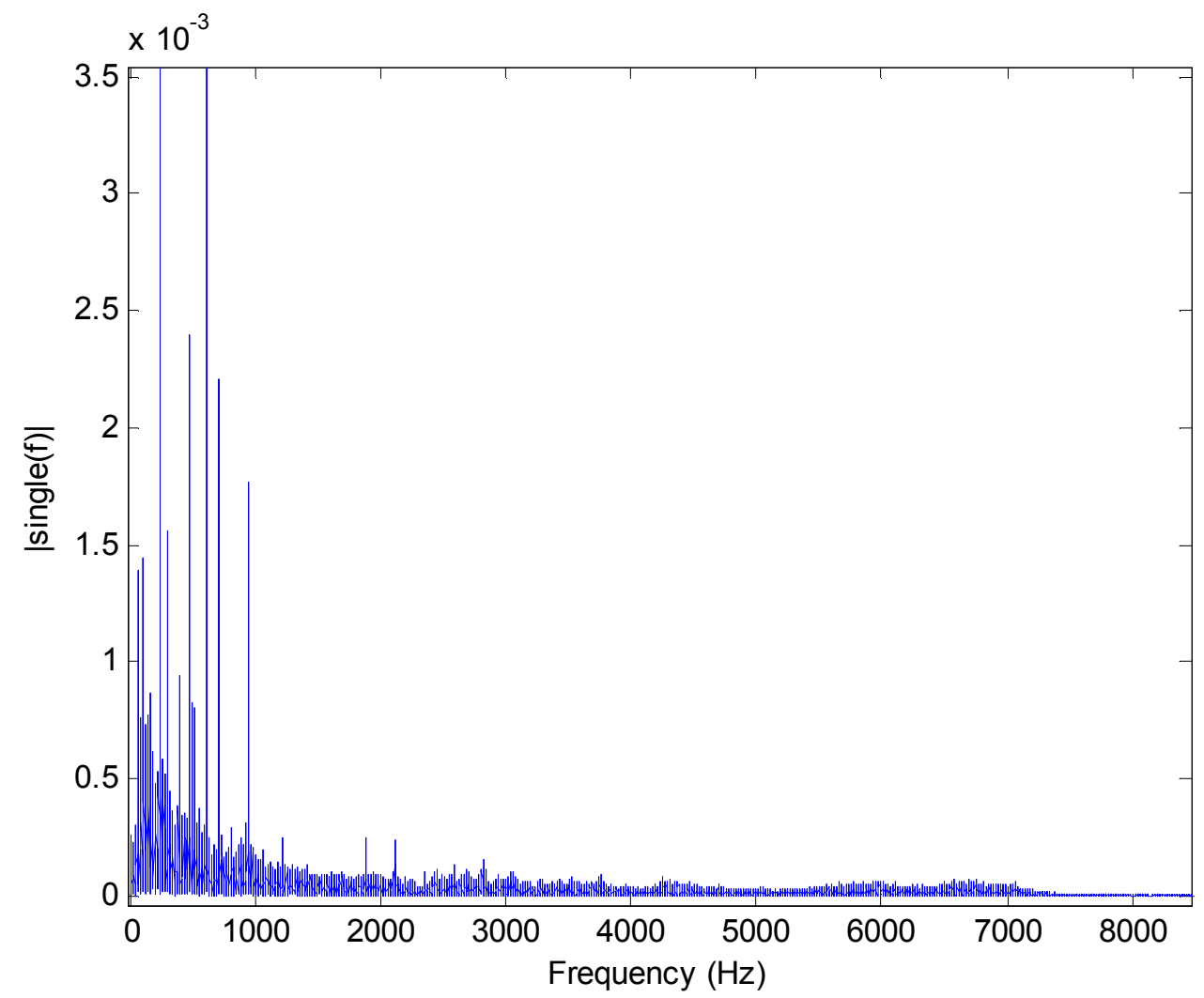

Figure 2. Frequency spectrum after process.

According to discrete signal Fourier expansion features, needing to low-pass signal filtering. A 4-order, low-pass filter with a normalized cut-off frequency of 0.95 is designed. [21] Figure 2 shows frequency spectrum after process. Compared with noise spectrum of air-conditioning running in [7], it can be judged that the band with large amplitude in the spectrum is the equipment running noise band.

\subsection{Running Noise Segmentation Processing and Filtering}

Equipment operating noise generated by different principles, so noisy band is different. As can be seen from Figure 2, the spectrum of background noise is concentrated below $1000 \mathrm{~Hz}$, and spectrum above $1000 \mathrm{~Hz}$ is flat. According to the above analysis band stop filter is used to filter out larger 
frequency components aiming to eliminate the operation noise. However, it is difficult to design low-band notch filters (band-stop filters) at high sampling rates. Due to low-frequency bands may also have fault sound sources, so it can not completely filter low frequency band even though contain more noise signals. Use frequency segmentation analysis in the frequency domain: Firstly, adopting a cut-off frequency of $1000 \mathrm{~Hz}$ low-pass filter process signal, there is no spectral aliasing after processed signal, then signal is down sampled to $3000 \mathrm{~Hz}$. Finally, designing corresponding frequency notch filter or band-pass filter aim to filter out some the larger amplitude band noise, the remaining band sound can achieve fault source positioning. Notch filter design is not completely ideal, surrounding band will have attenuation distortion, TDE algorithm is based on the correlation between signals, although some bands have attenuation, Robustness TDE algorithm can improve accuracy of estimate. Similarly, the same frequency domain preprocess above $1500 \mathrm{~Hz}$.

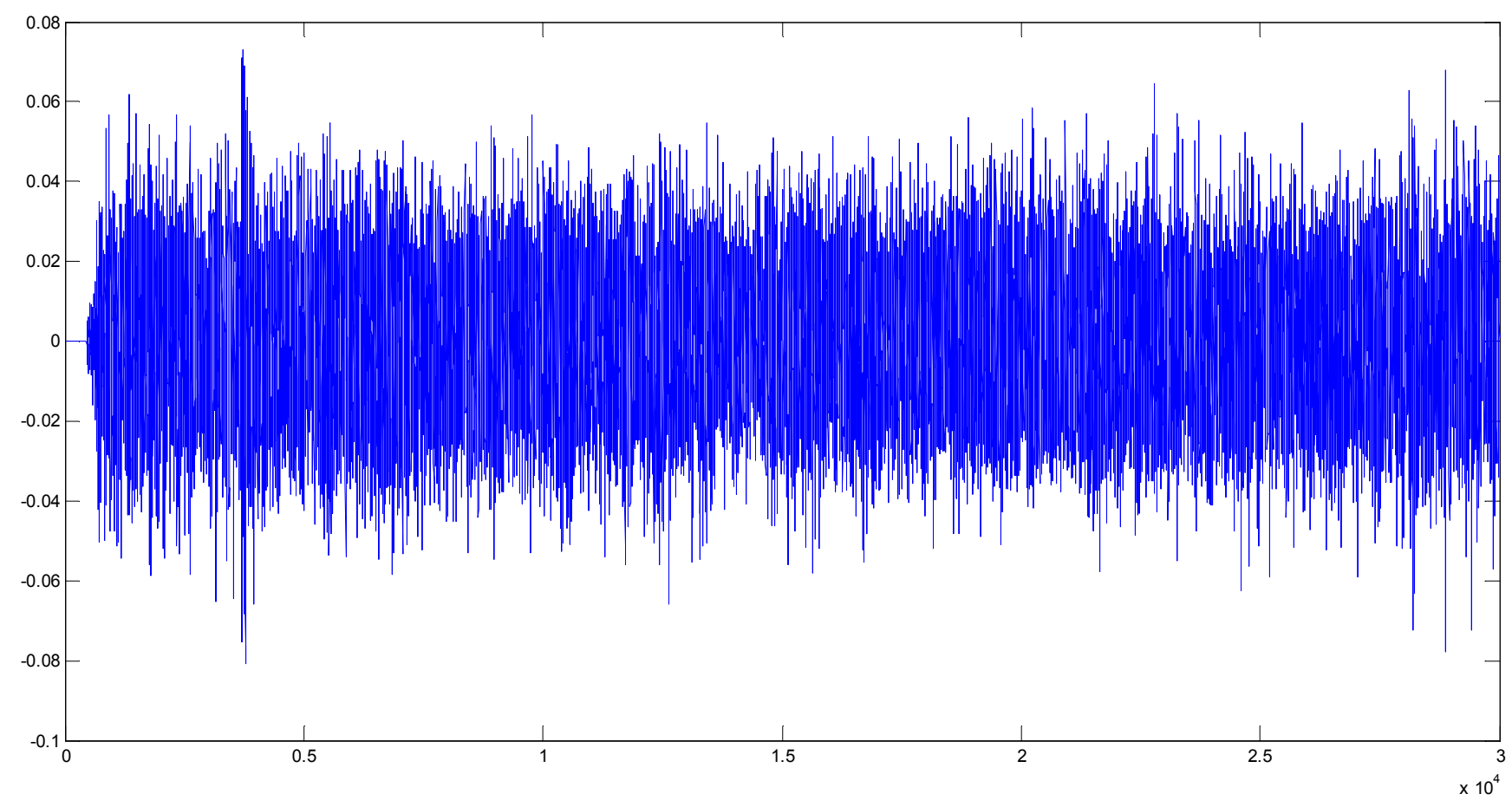

Figure 3. Time-domain waveform after process.

As shown in Figure 3 is the signal through the cut-off frequency of 0.06 12-order low-pass filter and down-sampling time-domain waveform, The signal can still monitor the existence of fan noise after processing.

There are amplitude frequency diagram and power diagram below frequency of $1500 \mathrm{~Hz}$ of the signal in Figure 4 (a) and (b), larger amplitude or power band in them are the frequency band of air conditioner running noise. Using Butterworth band-stop filter eliminate the impact of running noise on fault sound source localization. Filter design is based on the corresponding parameters of spectrum. Parameters such as Table 1, sampling frequency is $3000 \mathrm{~Hz}$, normalized frequency.

According to filter parameters, designing different frequencies band-stop filter in series filter the signal and calculate amplitude and power maps, as shown in Figure 8 (c)and(d), then, comparing the four pictures. Listen to the original signal and processed signal for comparison, after processing signal rotation noise elimination.

Table 1. Filter (notch) parameters.

\begin{tabular}{|c|c|c|c|c|c|c|c|}
\hline \multirow{2}{*}{$\begin{array}{l}\text { Band stop filter range } \\
62.25 \mathrm{~Hz} \text { notch }\end{array}$} & \multicolumn{2}{|c|}{$\begin{array}{l}\text { Pass band upper and } \\
\text { lower limits }\end{array}$} & \multicolumn{2}{|c|}{$\begin{array}{l}\text { Stop band upper and } \\
\text { lower limits }\end{array}$} & \multirow{2}{*}{$\begin{array}{l}\text { Passband maximum } \\
\text { attenuation(dB) } \\
0.1\end{array}$} & \multirow{2}{*}{$\begin{array}{l}\text { Stopband minimum } \\
\text { attenuation(dB) } \\
15\end{array}$} & \multirow{2}{*}{$\begin{array}{l}\text { Filter } \\
\text { order }\end{array}$} \\
\hline & 0.040 & 0.043 & 0.041 & 0.042 & & & \\
\hline $99.98 \mathrm{~Hz}$ notch & 0.065 & 0.068 & 0.066 & 0.067 & 0.1 & 15 & 4 \\
\hline $230 \mathrm{~Hz}-240 \mathrm{~Hz}$ & 0.15 & 0.163 & 0.153 & 0.16 & 0.2 & 10 & 5 \\
\hline $399.8 \mathrm{~Hz}$ notch & 0.198 & 0.201 & 0.199 & 0.200 & 0.1 & 30 & 5 \\
\hline 399.7Hz notch & 0.265 & 0.268 & 0.2663 & 0.267 & 0.1 & 40 & 5 \\
\hline $465 \mathrm{~Hz}-471 \mathrm{~Hz}$ & 0.309 & 0.316 & 0.310 & 0.314 & 0.2 & 8 & 6 \\
\hline 499.7Hz notch & 0.332 & 0.335 & 0.333 & 0.334 & 0.1 & 30 & 5 \\
\hline $510 \mathrm{~Hz}$ notch & 0.339 & 0.342 & 0.34 & 0.341 & 0.1 & 30 & 5 \\
\hline $607 \mathrm{~Hz}-612 \mathrm{~Hz}$ & 0.404 & 0.41 & 0.405 & 0.408 & 0.2 & 10 & 6 \\
\hline $705 \mathrm{~Hz}-711 \mathrm{~Hz}$ & 0.467 & 0.476 & 0.470 & 0.474 & 0.4 & 20 & 6 \\
\hline $936 \mathrm{~Hz}-942 \mathrm{~Hz}$ & 0.622 & 0.629 & 0.624 & 0.628 & 0.4 & 10 & 6 \\
\hline
\end{tabular}



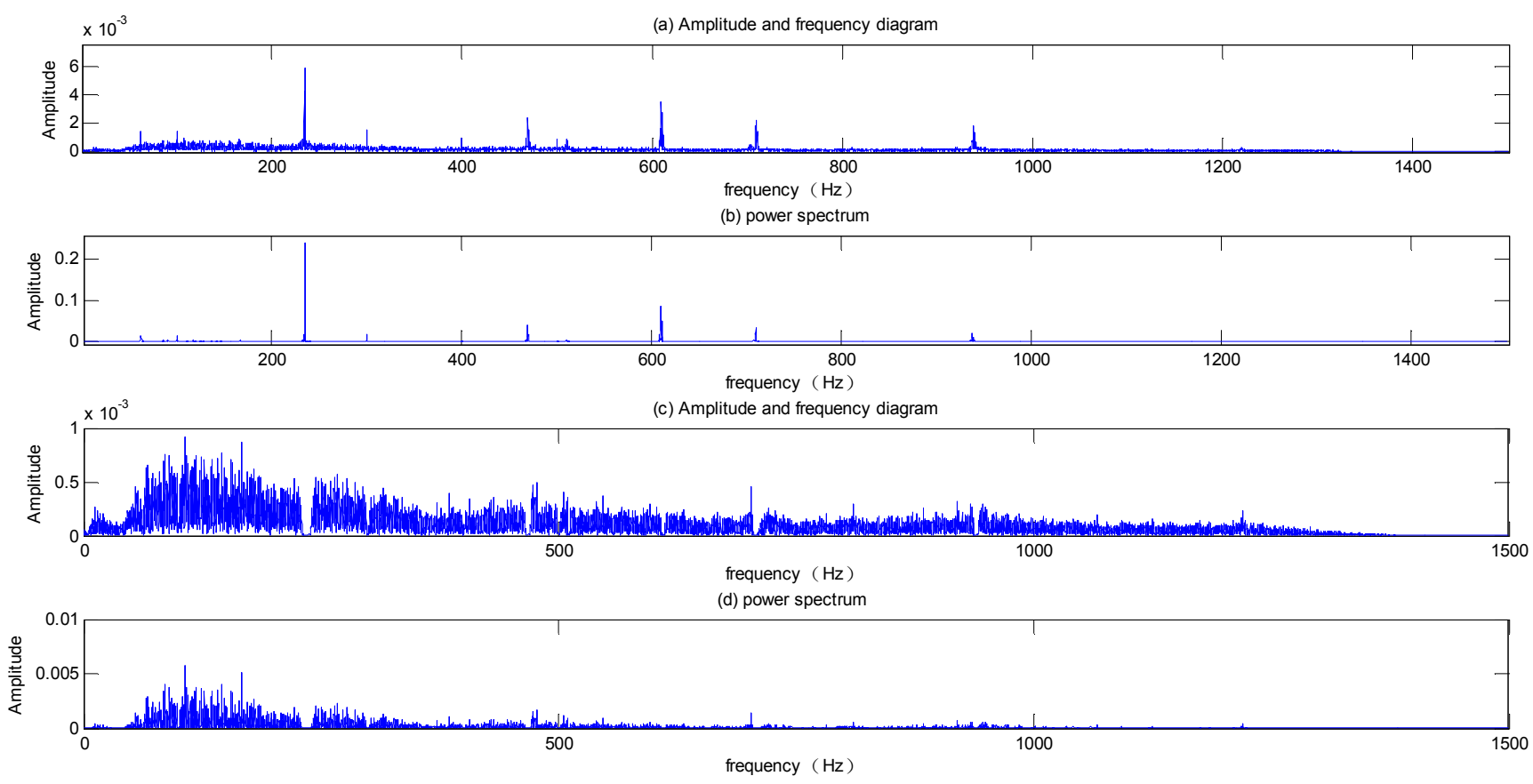

Figure 4. Filter processing amplitude and power chart comparison.

\subsection{Fault Sound SNR}

The sound level meter can measure space environment SNR. Its SNR is calculated by multiplying base 10 logarithm of ratio of sound power to reference sound power. In the case of noise source is not obvious or eliminating operating noise by covering, mobile phone software measure environment SNR during air conditioner operation and determine it within a certain range, then, simulating fault sound source in a better muffled environment. Obtained two SNR can get the fault signal SNR through corresponding calculation, it also offsets reference power. The sound level meter simulates the auditory sense of the human ear, with a weighted network, is a subjective measurement instrument, but also provides a basis for the measurement of SNR. In the air conditioner normal work, the software measured environment SNR around 60dB. (Running noise source is weakly distinguishable).

\section{Conclusion}

Analyze electrical equipment background noise, eliminate the influence of running noise, this can achieve locate the fault sound source in a specific frequency band; In the design of digital band-stop filter, the parameter selection refers to the existing filter parameter setting, which can filter out the target band noise. By adjusting the parameters, a better digital filter can be designed;

Using $\alpha$ distribution to model environment noise, it can simulate ambient noise with different pulse intensities according situation, which can model the ambient noise more accurately; the environment SNR is great importance to accurate modeling fault sound source signals and improve of TDE algorithm.

\section{References}

[1] A. F. Browne, S. Patel, S. A. Kaoti, S. M. Lopez and P. Parikh, Area acoustic and electromagnetic emissions monitoring of 3-phase motors, 2017 IEEE International Electric Machines and Drives Conference (IEMDC), Miami, FL, 2017, 1-6.

[2] Fredric Ottermo, Erik Möllerström, Anders Nordborg, Jonny Hylander, Hans Bern- hoff. Location of aerodynamic noise sources from a $200 \mathrm{~kW}$ vertical-axis wind turbine [J]. Journal of Sound and Vibration, 2017, 400.

[3] AN Lian-qu, FENG Qiang, SHEN Guo-qing, JIANG Gen-shan, ZHANG Shi-ping, WANG Peng. Power Source Passive Positioning Method for Utility Boilers Based on Multi-delay Identification [J]. Chinese Journal of Power Engineering, 2015, 35 (10): 798-804.

[4] Zhang Liang, Liu Zhiguang, Xiao Yanfan, et al. Design of sound source localization system for electromechanical equipment $[\mathrm{J}]$. Instrumentation Technology and Sensor, 2016 (2): 49-52.

[5] Shind e M P, Gupta S N. Signal detection in the presence of atmospheric noise in tropics. IEEE Trans. Communications, 1974, 22(8): 1055-1063.

[6] Georgiou P G, Tsakalides P, Kyriakakis C. Alpha-stable modeling of noise and robusttime-delay estimation in the presence of impulsive noise. IEEE Trans. on Multimedia, 1999, 1(3): 291-301.

[7] WANG Peng, ZHANG Xiao-tong, XU Li-yuan, HE Jie, XU Jin-wu. An indoor near-field measurement algorithm based on adaptive delay estimation [J]. Acta Chimica Sinica, 2017,40 (08): 1902-1917.

[8] $\mathrm{Xu}$ Chengwei. Study on the origin, diagnosis and noise reduction of air conditioning noise $[\mathrm{J}]$. Science and Technology Wind, 2012 (04): 108-109. 
[9] Qian Shiyu. Acoustic Camera - Making our Community Quiet [J]. Foreign Electronic Measurement Technique, 2009, 28 (02): 5-8.

[10] Li Zhiyuan, Chen Hui, Chen Pin, Lu Yimin. An acoustic power level sound intensity measurement method for large equipment [J]. VIBRATION. Testing and Diagnosticaion, 2013, 33 (02): 311-314.

[11] Junhao Li. Performance Comparison on Three Time Delay Estimation Algorithms Using Experiments [J]. Communications, 2017: 5.

[12] Jiang Rui, Li Qin, Zhong Jianwei. Analysis of power noise in distributed photovoltaic power station monitoring system [J]. Journal of Hubei Institute for Nationalities (Natural Sciences Edition), 2017, 35 (02): 219-223.

[13] Feller W. An introduction to probability theory and its applications. New York: John Wiley \& Sons Inc, 1966.

[14] HE Ji-Ai, CHEN Xing. Parameter Estimation of Communication Signals and Its Development [J]. finding Technology, 2017, 36 (3): 6-10.

[15] LIU Wen-hong, ZHANG Shuai, QIU Tian-shuang. Comparison of $\alpha$-stable distribution feature index estimation methods [J]. Journal of Shanghai Dianji University, 2013, 16 (04): 183-187.
[16] Guan Jiashi, Shi Daowu, Qiu Jianwen, Shi Hongwei. A Method to Estimate the Stable Distribution Symmetry Coefficient and Dispersion Coefficient [J]. Zhonghua University of Science and Technology (Natural Science Edition), 2017, 45 (09): 23-27.

[17] Nikias C L, Shao M. Signal processing with Alpha-stable distributions. New York: John Wiley \& Sons Inc, 1995.

[18] Liu Wenhong. Estimation method and application of time delay under impulsive noise [D]. Dalian University of Technology, 2007.

[19] A. Chandra, Measurements of radio impulsive noise from various sources in an indoor environment at $900 \mathrm{MHz}$ and $1800 \mathrm{MHz}$, The 13th IEEE International Symposium on Personal, Indoor and Mobile Radio Communications, 2002, 639-643 vol. 2.

[20] Shen Qingyue. Selection of sampling rate and quantization bit depth in audio work [J]. Bound Sound Technology, 2013 (02): $54-55+60$.

[21] Shen Zaiyang. MATLAB Signal Processing [M]. Beijing: Tsinghua University Press, 2017: 234-258. 